\title{
EVALUACIÓN DE INTERVENCIONES SANITARIAS Y SOCIALES: MIDIENDO LOS RESULTADOS PARA ORIENTAR LA TOMA DE DECISIONES
}

\author{
EVALUATION OF SOCIAL AND SANITARY INTERVENTIONS: \\ APPRAISING THE RESULTS TO GUIDE DECISIONS
}

\author{
Alonso Soto $0^{1, a, b}$
}

El concepto de medicina basada en evidencias es ampliamente conocido y aplicado en las ciencias de la salud como un modelo para la toma de decisiones. Preconizada inicialmente en base a un enfoque individual, su filosofía ha sido incorporada a la salud pública a efectos de guiar las decisiones sanitarias. La medicina basada en evidencias en salud pública se sustenta así, en la evaluación objetiva de las intervenciones a efectos de cuantificar su impacto real y decidir la conveniencia de su implementación de manera general y sostenible tomando en consideración a los sistemas de información y a los modelos de planeamiento existentes ${ }^{(1)}$. Sin embargo, la toma de decisiones a partir de la mejor evidencia disponible no debe ser patrimonio de las ciencias de la salud y debería ser una constante en la mayoría de intervenciones destinadas a mejorar el bienestar de la población.

Tradicionalmente hemos asumido que el beneficio de los programas sociales de intervención en salud, educación y ambiente, es incuestionable. Sin embargo, cuando revisamos el sustento científico de estas políticas vemos que muchas de ellas se han basado en juicio de expertos y recomendaciones de cooperantes internacionales. De hecho, nadie pondría en tela de juicio la implementación -por citar un ejemplode la dotación de alimentos a personas en desventaja social. Pero, como es conocido, las dificultades de implementación pueden hacer que el impacto de esta política no sea necesariamente el esperado tal como ha ocurrido con el impacto nutricional del Programa del Vaso de Leche el que, pese a su gran alcance, no ha logrado disminuir las tasas de desnutrición de los beneficiarios ${ }^{(2)}$. Es por esto que resulta imprescindible realizar un análisis científico exhaustivo inicial en el momento de la planificación de las intervenciones, para seleccionar las mejores; realizar estudios de líneas de base; un análisis interino que permita corregir errores, realizar mejoras e identificar los cuellos de botella de estos programas y una evaluación final para determinar su real impacto. Sorprendentemente, la implementación de programas sin un mecanismo claro y preespecificado de evaluación o sin siquiera una línea de base claramente establecida, parece ser la regla más que la excepción. Las mejoras en los indicadores sociales y de salud pueden corresponder simplemente al resultado de la evolución natural asociada a los cambios en la condición socioeconómica más que a un programa en particular; los indicadores utilizados en las evaluaciones no son estáticos y las condiciones de vida de una población pueden mejorar o no, independientemente de los programas de apoyo. Por otro lado, si entre una multitud de posibles indicadores, se escoge a posteriori aquellos que muestren mejores cifras, estas pueden ser susceptibles de manipulación, otorgando la falsa imagen de eficiencia en intervenciones ineficientes.

Como en cualquier disciplina, la medición del impacto de un programa es imprescindible para determinar su efectividad real. Evidentemente, se va a encontrar resistencia a ello, pues la pregunta del impacto puede tener como respuesta la ausencia de este impacto o, peor aun, un efecto negativo, lo cual puede interpretarse como un riesgo político innecesario. Sin embargo, nosotros creemos que la demostración científicamente válida de un impacto positivo de los programas de salud será crucial no solo para garantizar su sostenibilidad ante cambios de autoridades o crisis económicas, sino además para fortalecer el apoyo por los cooperantes externos e internos involucrados y la participación de la comunidad.

\footnotetext{
Hospital Nacional Hipólito Unanue, Lima, Perú.

a Editor Científico. Revista Peruana de Medicina Experimental y Salud Pública.

b Médico internista, Doctor en Medicina
} 
Existen otros beneficios de incorporar los métodos de evaluación científica a la implementación de los programas sociales y de salud. Por ejemplo, uno de los riesgos potenciales de la implementación de cualquier programa de beneficios sociales es la corrupción, especialmente en programas que incluyan la distribución de alimentos o dinero y que no tengan establecidas medidas de seguridad que certifiquen que los beneficios lleguen a quienes realmente los necesitan. Las mediciones de impacto basadas en un protocolo científico claramente establecido y con monitoreo de indicadores de proceso y resultado son herramientas útiles para garantizar la ejecución óptima de un programa y disminuir así la posibilidad de actos corruptos. Sin embargo, estas deben tomarse como lo que son: parámetros que ayudan a la evaluación. La falta de resultados positivos debe motivar acciones para identificar las causas tanto inherentes a la intervención como a factores externos que puedan obstaculizar su adecuada implementación. Igualmente, en aquellos programas con resultados positivos, deben identificarse aquellos factores que han contribuido al éxito a efectos de tenerlos en cuenta para generar políticas a futuro.

Existen distintas metodologías para poder cuantificar el impacto de una intervención; sin embargo, uno de los aspectos principales que se deben tomar en consideración es el uso de controles. Es decir, grupos comparables en los que no se realice la intervención. La primera dificultad que afronta la evaluación de programas plausiblemente beneficiosos desde el punto de vista ético es si resulta posible brindar un beneficio a ciertos grupos y no a otros. Ello evidentemente depende de la percepción de la utilidad de la intervención. Si existe realmente incertidumbre sobre el beneficio, resulta razonable -con el consentimiento y participación de las autoridades locales- el comparar las intervenciones en ensayos de campo u otro tipo de estudio para grupos poblacionales. En casos de intervenciones en las que existe consenso en que deberían ser beneficiosas, existen alternativas interesantes como los diseños de ensayos escalonados en los que se avalúa la implementación progresiva de un programa ${ }^{(3)}$ y pese a las críticas conceptuales como modelo metodológico, la evaluación cuasi experimental (4) no deja de ser una posibilidad que, si bien es cierto, resulta más susceptible a sesgos que diseños claramente experimentales, es una alternativa que puede brindar información importante en particular para intervenciones complejas ${ }^{(5)}$.
La investigación es sin lugar a dudas no solo un reflejo del desarrollo de una nación sino la vía para alcanzarlo. En ese sentido, la investigación en salud no solo incluye a las ciencias básicas sino también al estudio y decisión final sobre intervenciones sanitarias que se considere implementar. Tal vez el mejor ejemplo de la importancia de instituciones generadoras de bases científicas para las decisiones políticas es el Instituto Nacional de Salud y Excelencia Clínica del Reino Unido (http://www.nice. org.uk/)

Compartimos la ideología del enfoque a la salud desde el punto de vista de la equidad y hemos seguido con expectativa los ofrecimientos del actual gobierno al impulso de la ciencia y tecnología. Por lo que auguramos que la nueva gestión gubernamental tendrá en consideración esta corriente de intervenciones basadas en evidencias y el contexto en el que se desarrollan los fenómenos de salud. Es momento de aprender de nuestros errores y aciertos, y de tomar las decisiones no solo con criterios políticos o coyunturales sino en base a procesos definidos que incluyan el análisis de la mejor evidencia disponible incluyendo experiencias nacionales y extranjeras y, una vez tomadas las decisiones, analizar con metodologías claras su desarrollo e impacto, evaluando críticamente los resultados para la mejora continua de nuestras decisiones. Ello redundará en beneficio de la población y será un elemento trascendental si queremos un sistema de salud eficiente, equitativo y justo.

\section{REFERENCIAS BIBLIOGRÁFICAS}

1. Brownson R, Baker A, Leet T, Gillespie K (Editors). Evidence-Based Public Health. New York: Oxford University Press; 2003.

2. Gajate G, Inurritegui M. El impacto del vaso de leche sobre el nivel de nutrición infantil. Economía y Sociedad. 2003;50:63-70.

3. Brown C, Lilford R. The stepped wedge trial design: a systematic review. BMC Medical Research Methodology [Internet]. 2006[citado el 12 de agosto del 2011];6(54):[9 p.]. Disponible en: http://www.biomedcentral.com/content/ pdf/1471-2288-6-54.pdf. doi:10.1186/1471-2288-6-54.

4. Campbell D, Stanley J. Experimental and Quasi-Experimental Designs for Research. New York: Houghton \& Mifflin Co.; 1966.

5. Murray DM, Pennell M, Rhoda D, Hade EM, Paskett ED. Designing Studies That Would Address the Multilayered Nature of Health Care. J Natl Cancer Inst Monogr. 2010;2010(40):90-6. 